\title{
A Study On How Women Balances Job and Family in the Wake of Welfare Room
}

\author{
Abhishikta Pramanik ${ }^{1}$, Mounika Veeragandham ${ }^{2 *}$, Suman Gundu ${ }^{3}$ \\ ${ }^{1,2}$ Student, Universal Business School, Karjat, India \\ ${ }^{3}$ Professor, Universal Business School, Karjat, India \\ *Corresponding author: mounikaveeragandham15@gmail.com
}

\begin{abstract}
Work life balance majorly depends upon the way we prioritize our tasks and execute them. In current day technologybased ecosystem with the tremendous pressure by employers on workforce resulted in loss of significance of work life balance. Work life balance is a vital aspect of a healthy work environment. (Alankohil, 2018). The imbalance of professional and personal lives seriously impacts the individual, which may sometimes lead to chronic stress and mental depression. In this paper we highlight the challenges associated in managing the work life balance.
\end{abstract}

Keywords: Chronic stress, Mental depression, Technology, Work environment, Work life balance.

\section{Introduction}

In today's fast paced world, the significance of work life balance is truly evolving in rapid pace but with a different approach. In recent times we are visualizing many instances related to employees claiming high end pressure, working on weekends and issues with management. (Joe Wedgwood, 2019). These are few ways where it disturbs the balance and hampers the balance between professional and personal life. (TalentIntelligence, 2019). Globally top executives provided few inputs such as setting up our priorities, understanding and being aware of a thin line between professional and personal bench and last but not least dedicating sometime for self. Interestingly the approach towards work life balance is emerging in this paper we clearly reflect the insights on the variance of work life balance in men and women. Implementing these results in good striking work life balance. (Rashid Nawaz, 2019). Irrespective of gender equality the ratio of work family conflict is very huge. (Economic Times, 2017). In this research paper we clearly provide the insights on what factors influences work life imbalance and also shed light on the approaches that can be implemented.

\section{A. Research Objectives}

- To study the major consequences of poor work life balance.

- To evaluate the factors responsible for the work life imbalance for women.

- To understand the approaches that can be implemented at workplace to maintain good work life balance.

\section{B. Hypothesis}

1) Current day jobs have critical impact on work life balance.

2) Women workforce facing crucial challenges in managing work life balance.

\section{Research Methodology and Data Analysis}

As part of our research study, we conducted both primary and secondary research to provide insights upon understanding how women deals with the hiccups in workplace and at the same time how women ensures the work life balance. Accordingly, to draw these final conclusions various Newspapers, company blogs, official sites, executive insights, and articles of leading consulting firms has been taken into consideration. Primary research was carried out to know the vital attributes responsible in achieving a healthy work life balance. It was done by conducting a survey through online questionnaire.

To achieve the mentioned objective, we had the following research design. The sample size includes 100 respondents of working professional's woman ranging from the age group of 21 to age group of 58. In terms of segmentation I chosen Age, Gender, and education as my segmentation variables. Moreover, to pursue detailed analysis we made use of various tools such as bar graphs, pie charts and line charts with the obtained responses. The methodology of research is purely based on the facts and insights that we collected through secondary research and the responses we received through the questionnaire as part of primary research.

Profile of the target sample: We included males and females of the age group ranging from 21 to the age group up to 60, where the sample size comprises of graduates, postgraduates, working professionals and Business owners.

Source of data:

Primary research: sample size of 100

Secondary research: Newspapers, research papers, blogs, and articles by few consulting firms are considered.

Period of the study: The study was conducted for a month as part of learning experience in the month of July and August.

\section{Literature Review}

Executives opine the emerging need of fostering the sense to 
boost the morale of the employee. In other words, employees needs to schedule flexibility so that there won't be a chances of potential burnout and anxiety. To mitigate such roadblocks employee assistance programs and financial assistance are one of the options that can be relied on. Furthermore, Fortune magazine opine addressing issues related to work life balance is top priority Moreover, being disconnected with the social group also leads to work life imbalance. (Livemint, 2020).

Jeff Bezos opines extremely different concept when compared to his counterparts in terms of work life balance. He says instead of work life balance it is important to have work life harmony. The core reason behind opting this approach is it positively impacts and helps to prioritize our well-being. In addition to this $40 \%$ of the work force opines they are not provided with enough work life balance. Globally the issues associated to work life are increasing on the leader's radar day to day. (sarahBerger, 2018).

Chief executives of the global firm opine it's important to promote gender equality and its extremely imperative and linked to performance of the resource. Clear direction needs to be provided to employees so that they can be aware of organizational focus and can clearly set their goals. A streamlined strategy needs to be formulated so that the diversity among workforce is maintained. (Mckinsey \& Company, 2015)

\section{Secondary Research}

Executive insights from medium magazines provides few fascinating insights on how women can manage work life balance. At first place we need to master at sorting our priorities , post that we need to maintain transparency and honesty in order to confront the flaws in the system if any for holistic development of the organization.in addition to limiting our distractions we also needs to practice on how to delegate our opinion. (vartikakashyap, 2018)

Mckinsey opines women are facing challenges in the age of automation. To weather this disruption several scenarios and job creation trends needs to be referred to understand the locus of possible outcomes (The future of women at work: Transitions in the age of automation, 2019). Sometimes social attitudes around impacts the role of women which results in preferring a male candidate for the same. Though our law provides equal opportunity, employers also need to facilitate this provision for the women workforce. (Seemavarma, 2016)

According to PWC small firms are setting things right. As per their survey they came to know that majority of the segment related to baby boomers voiced their opinion stating they have a committed partnership with employers. In other words, this often reflects the great deal of trust. Big firms trying to coach their employees about the importance of gratitude and the core purpose behind employee ranks. This is because autonomy and appreciation are two important aspects which complements the companies leadership. As per the reports majority of the people opine work life balance and having a meaningful work is extremely important. (PWC, 2016).

\section{Primary Research}

1) Please specify your age group

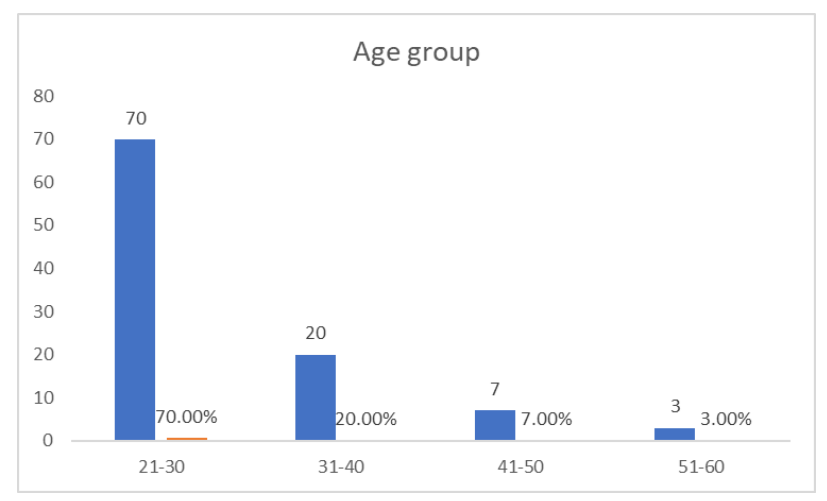

According to the data majority of the respondents are in the age group of 21-30 followed by 31-40.

2) Please specify your Gender

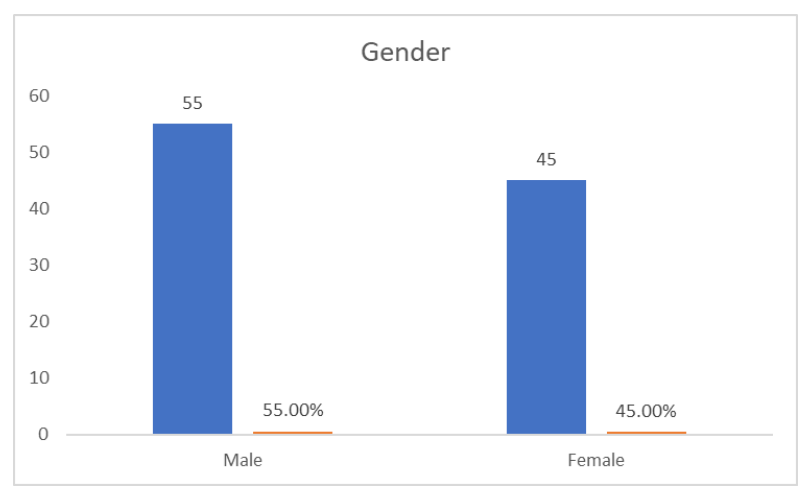

$55 \%$ of male express their opinions on women work life balance majority followed by females with $45 \%$. The ratio of respondents are almost close in terms of percentages. Both groups have voiced their opinion.

\section{3) Please specify your academic/professional qualification}

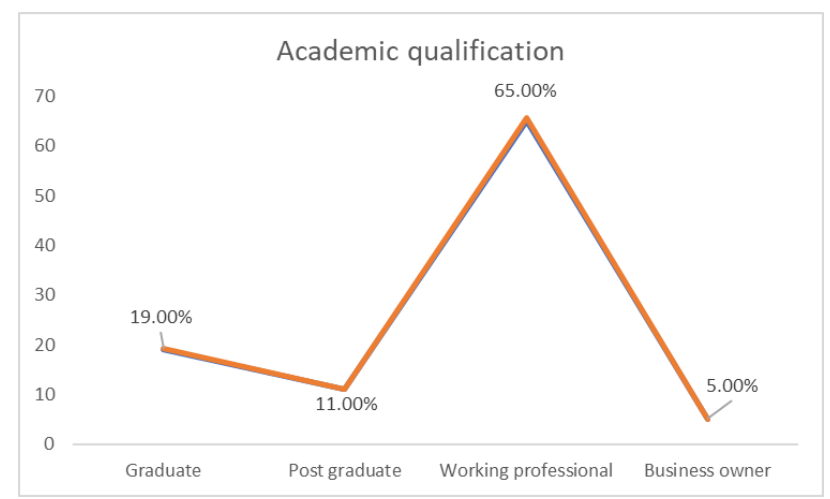

Based on the data most of the respondents comes under segment of working professionals followed by graduates and 


\section{IJRESM journals.resaim.com/ijresm | ISSN (Online): 2581-5792 | RESAIM Publishing}

postgraduates. Interestingly even business owners also provided their valuable opinion.

4) Please specify which sector do you belong to

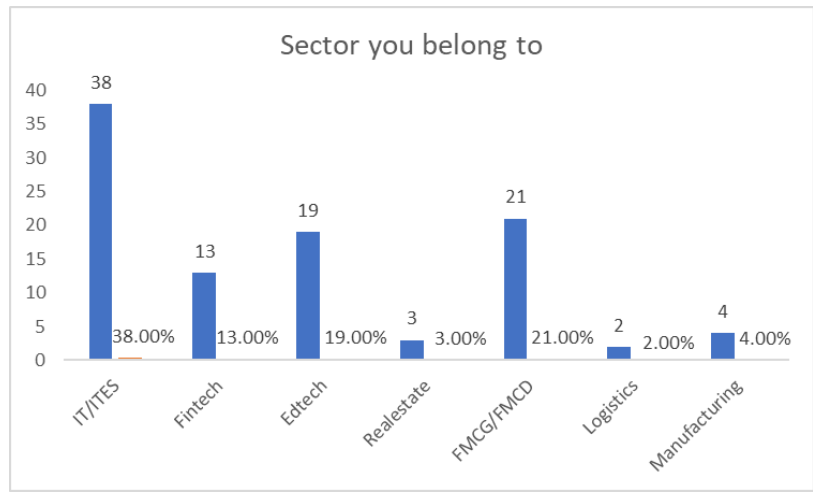

According to the data respondents are from IT/ITES followed by FMCG/FMCD sector. Respondents related to manufacturing and logistics are low compared to other sectors.

5) Which of the below do you think are the major consequences for poor work life balance

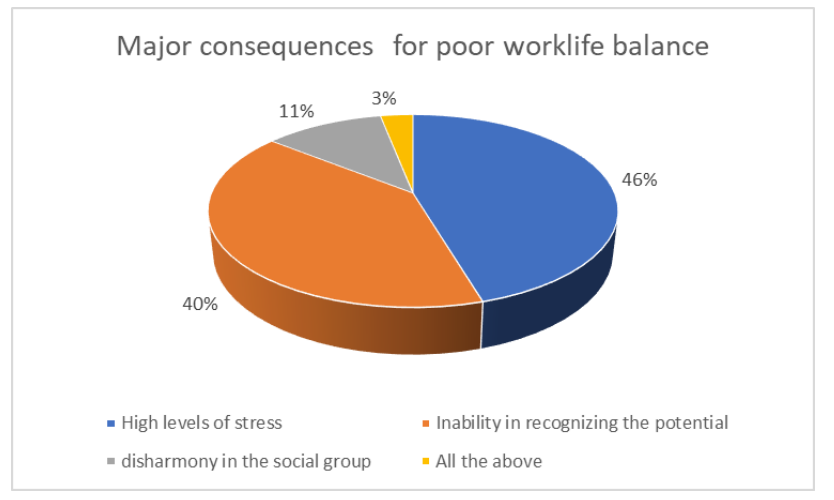

According to the data, most of the respondents opine inability in recognizing the potential followed by high levels of stress are the core reasons for the poor work life balance.

6) Which of the below do you think are the critical challenges faced by women at workplace

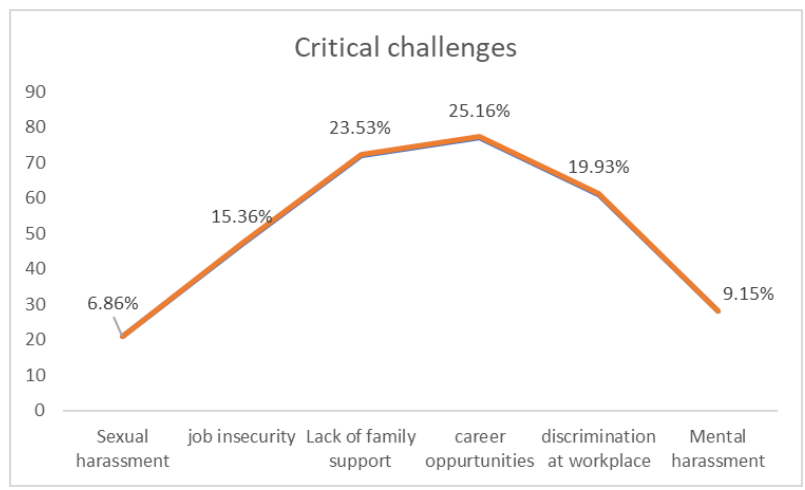

Based on the data lack of career opportunities, family support followed by discrimination at workplace are the potential challenges faced by women at workplace.in few other cases even sexual and mental harassments are also taking place on women.

7) Which of the below factors according to you are responsible for a work life imbalance in women

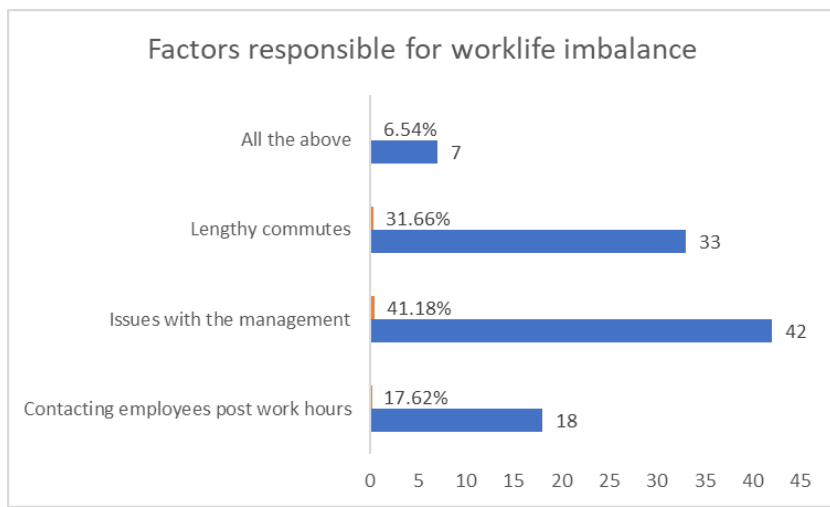

Most of the respondents opine Issues with the management and lengthy commutes followed by contacting employees post work hours are the important factors responsible for work life imbalance.

8) Which of the below parameters impact quality of work life

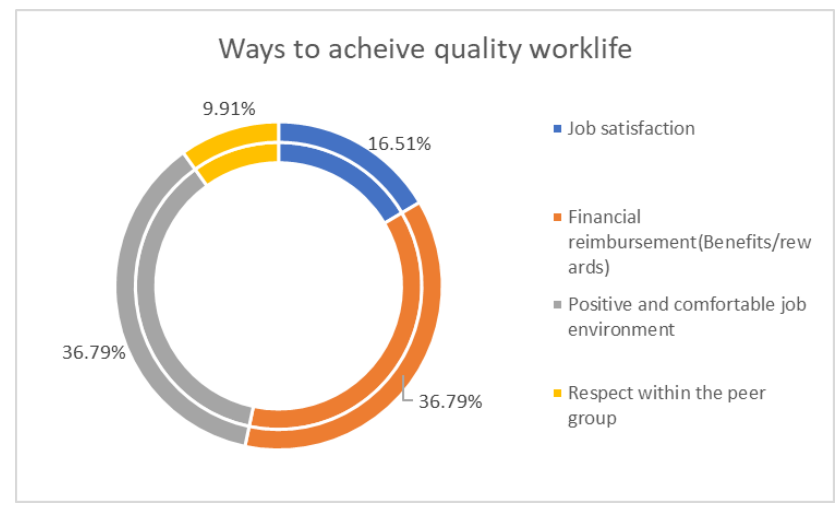

Based on the report above, rewarding employees, providing benefits for the efforts invested are one of the parameters which enhances quality of work life. Job satisfaction and work environment termed as secondary as per the data in terms of achieving good balance in work life. 
9) What approaches do you think helps to maintain good work life balance

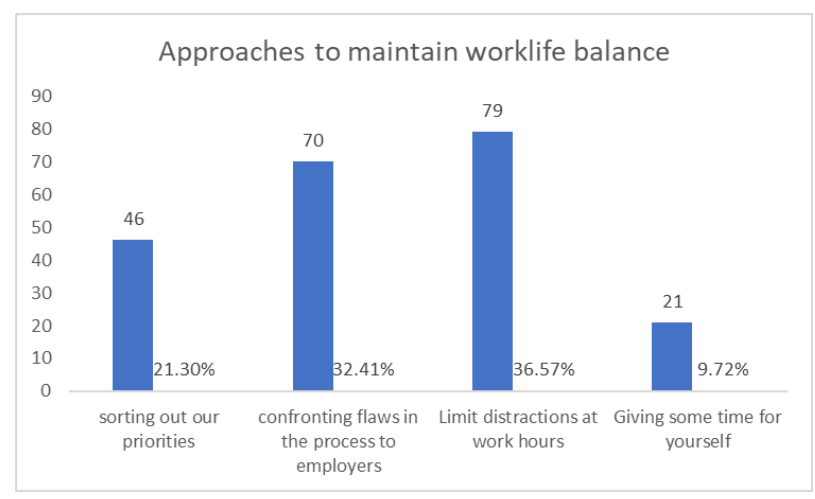

Limiting distractions at workhours and confronting flaws in the process to the employer helps every professional to maintain work life imbalance. Setting out our priorities is also one of the important attribute that we need to practice in order to lighten our stress and execute tasks with more accuracy.

10) Which of the below ways can you suggest implementing at the workplace to encourage health work life balance

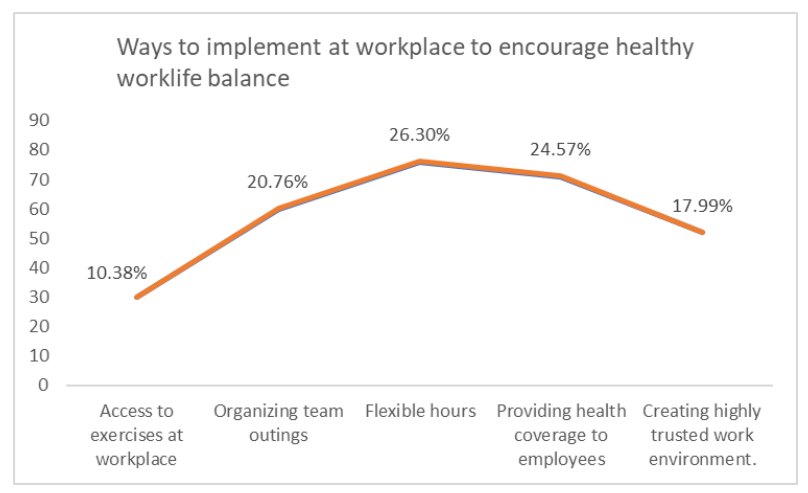

According to the data majority of the people opine flexible workhours and providing health coverage to employees followed by organizing team outings are one of the useful ways that can be implemented at workplace to encourage healthy work life balance.

\section{Conclusion}

Based on all the responses provided by postgraduates, graduates, professionals and business owners, we depicted few interesting insights such as inability in recognizing the potential and high level of stress which sometimes occurs due to disharmony with social group are the major consequences for poor work life balance. Moreover, lengthy commutes and contacting employees post work hours are some instances where it leads to work life imbalance. Last but not least limiting distractions in worktime and organizing some team/company outings gives good mental relief and also helps to achieve quality work life balance. In majority of the cases women seems to manage this very aptly. This also got clearly reflected in the responses we received.

\section{References}

[1] Alankohil. (2018, March 27). The Evolving Definition of Work-Life Balance. Retrieved from Forbes:

https://www.forbes.com/sites/alankohl1/2018/03/27/the-evolvingdefinition-of-work-life-balance/\#5efb2c939ed3

[2] Economic Times. (2017, July 29). Retrieved from Economic Times: https://economictimes.indiatimes.com/magazines/panache/men-struggleas-much-as-women-to-maintain-work-lifebalance/articleshow/59823097.cms? from $=\mathrm{mdr}$

[3] Joe Wedgwood. (2019, August 29). Happiness Index. Retrieved from Happiness Index: https://thehappinessindex.com/employee-engagement/importance-ofwork-life-balance/

[4] Livemint. (2020, August 1). How the pandemic may change 'work-life balance' forever. Retrieved from Livemint: https://www.livemint.com/companies/people/how-the-pandemic-maychange-work-life-balance-forever-11596259134080.html

[5] Mckinsey \& Company, (2015, February 01), Gender balance and the link to performance. Retrieved from Mckinsey: https://www.mckinsey.com/featured-insights/leadership/gender-balanceand-the-link-to-performance

[6] Mckinsey \& Company. (2019, June 04). The future of women at work: Transitions in the age of automation. Retrieved from Mckinsey \& Company:

https://www.mckinsey.com/featured-insights/gender-equality/the-futureof-women-at-work-transitions-in-the-age-of-automation

[7] PWC. (2016, may). Work-life 3.0: Retrieved from PWC: https://www.pwc.com/ee/et/publications/pub/pwc-consumerintellgience-series-future-of-work-june-2016.pdf

[8] Rashid Nawaz. (2019, October 13). How a Working Woman Can Maintain Balance in Work and Life?

Retrieved from Thrive Global: https://thriveglobal.com/stories/how-aworking-woman-can-maintain-balance-in-work-and-life/

[9] Sarah Berger, (2018, August 9), Jeff Bezos doesn't like the idea of 'worklife balance' - here's what he swears by instead. Retrieved from CNBC: https://www.cnbc.com/2018/08/09/what-jeff-bezos-does-instead-ofwork-life-balance.html

[10] Seemavarma. (2016, December). Retrieved from https://www.krishisanskriti.org/vol_image/08Aug201809085914\%20\%2 $0 \% 20 \% 20 \% 20 \% 20 \% 20 \% 20 \% 20 \% 20$ Seema\%20Verma $\% 20 \% 20 \% 20 \% 2$ $0 \% 20 \% 20 \% 20 \% 20820-823$.pdf

[11] Talent Intelligence. (2019, October 2), 3 Factors that Affect Work-Life Balance. Retrieved from Talent Intelligence:

https://www.talentintelligence.com/blog/3-factors-that-affect-work-lifebalance

[12] The future of women at work: Transitions in the age of automation. (2019, June 4).

Retrieved from Mckinsey:

https://www.mckinsey.com/featured-insights/gender-equality/the-futureof-women-at-work-transitions-in-the-age-of-automation

[13] Vartikakashyap. (2018, August 27). A Working Woman's Guide to Maintain Work-life Balance. Retrieved from Medium: https://medium.com/thrive-global/a-working-womans-guide-tomaintain-work-life-balance-27aa63e2f743 